\title{
Contribuições do Modelo de Rasch para Procedimentos de Intervenção em Habilidades Sociais
}

\author{
José Maria Montiel - Centro Universitário Fundação Instituto de Ensino para Osasco, Osasco, Brasil \\ Daniel Bartholomeu - Centro Universitário Fundação Instituto de Ensino para Osasco, Osasco, Brasil
}

\begin{abstract}
Resumo
O presente estudo visou analisar se o modelo de Rasch pode facilitar o aprendizado de condutas socialmente hábeis em crianças. Foram realizados dois estudos, um para estabelecer pontos de corte e níveis de dificuldade numa medida de habilidades sociais e outro para analisar a intervenção baseada nessa hierarquia. Os resultados do Estudo 1 indicam que as condutas mais fáceis são as relacionadas à civilidade e altruísmo, basicamente e as mais difíceis as relacionadas à assertividade. No Estudo 2, os resultados da intervenção planejada com base no nível de dificuldade dos itens indicou a melhora das habilidades sociais das crianças no mesmo sentido do nível de dificuldade dos itens. De modo geral, o modelo de Rasch demonstrou eficácia como auxílio para analisar a dificuldade de execução das condutas sociais e no planejamento e implementação de intervenções nesta área.

Palavras-chave: intervenção, escola, psicometria, aprendizagem
\end{abstract}

Contributions of Rasch Model to Social Skills Intervention Procedures

\begin{abstract}
This study aimed to examine whether the Rasch model can facilitate the learning of socially skilled behavior in children. Two studies, one to establish cut points and levels of difficulty in measuring social skills and another to analyze the intervention based on this hierarchy, were performed. The Study 1 results indicate that the easiest behaviors are related to civility and Altruism, and the hardest related to assertiveness. In Study 2, the intervention results planned based on the level of difficulty of the items indicated the improving in social skills of children in the same direction as the items level of difficulty. In general, Rasch model was effective as an aid to assess the difficulty of social skills measures and to help planning and implementing interventions within this area.

Keywords: intervention, school, psychometrics, learning
\end{abstract}

Contribución del modelo de Rasch para Procedimientos de Intervención en Habilidades Sociales

\begin{abstract}
Resumen
Este estudio tuvo como objetivo analizar si el modelo de Rasch puede facilitar el aprendizaje de conductas socialmente hábiles en los niños. Se realizaron dos estudios, uno para establecer puntos de corte y niveles de dificultad para medir las habilidades sociales y otro para analizar la intervención basada en esta jerarquía. Los resultados del estudio 1 indican que las conductas más fáciles son las relacionadas con civismo y altruismo y las más difíciles son las relacionadas con asertividad. En el estudio 2 , los resultados de la intervención planificada, con base en el nivel de dificultad de los ítems, indicó la mejoría de las habilidades sociales de los niños en el mismo sentido del nivel de dificultad de los ítems. En general, el modelo de Rasch demostró eficacia como auxilio para analizar las dificultades de ejecución de las conductas sociales y para la planificación e implementación de intervenciones en esta área.

Palabras clave: intervención, escuela, psicometría, aprendizaje
\end{abstract}

A pesquisa sobre o treinamento de habilidades sociais no Brasil tem tido repercussões importantes em diferentes contextos. Tal afirmação é detalhada no estudo de Murta (2005) ao descrever que até 2005, 17 publicações nacionais descreveram o treinamento de habilidades sociais em diferentes contextos e populações, mas predominantemente com prevenção primária e secundária, com processos de avaliação verbais e técnicas observacionais em pais, crianças e professores, principalmente. A maior parte destas empregou delineamento pré-experimental com pré e pós-teste. Poucos empregaram delineamento quase experimental ou experimental. A literatura sobre intervenções com habilidades sociais no Brasil é bastante vasta e investigações com inúmeros contextos já foram feitas com pais, professores, universitários, adolescentes e crianças, dentre outros, como pesquisas relacionando dificuldades de aprendizagem ou deficiência intelectual (Molina \& Del Prette, 2006; Freitas \& Del Prette, 2010; Bartholomeu, Montiel, \& Pessotto, 2011; Del Prette, Barreto, \& Freitas, 2011) e alguns destes devem ser mais bem detalhados. Os trabalhos de (Bolsoni-Silva, Fabiane, \& Ribeiro, 2008; Bolsoni-Silva et al., 2008) são exemplos desses e focaram no treinamento de habilidades sociais educativas com pais de crianças com problema de comportamento, já que os déficits 
de habilidades sociais educativas dos pais tendem a afetar o comportamento de seus filhos. Os resultados evidenciaram melhoras nas habilidades sociais após a intervenção focada nas habilidades sociais educativas parentais, sendo que os pais melhoraram diversas destas, como expressar sentimentos positivos, agradecer elogios, dizer não e negociar limites.

Ainda com mães e cuidadoras, o estudo de Bolsoni-Silva, Silveira e Ribeiro, (2008) também objetivou a avaliação dos efeitos de um programa de intervenção para a promoção de habilidades educativas e prevenção de problemas de conduta em crianças. Os resultados do programa de intervenção foram positivos no sentido de que os pais aprenderam a remediar os problemas de comportamento infantis com redução de tais problemas sendo observado uma melhora nas habilidades sociais dos filhos, sobretudo em assertividade. As autoras sugerem que medidas de autorrelato na análise das condutas sociais das crianças são restritas, bem como enfatizam que a preocupação com a promoção de comportamentos socialmente habilidosos deva ser maior do que com a eliminação dos comportamentos problemáticos, o que é, para as autoras, um procedimento pouco citado na literatura. Nessa perspectiva, Pinheiro, Haase, Del Prette, Amarante e Del Prette (2006) descreveram em seu trabalho um programa de treinamento de habilidades sociais para pais de crianças com problemas de conduta. Os autores concluem que as habilidades sociais educativas podem contribuir positivamente para o desenvolvimento de práticas disciplinares não coercivas e que produzam resultados positivos sobre as condutas dos filhos. É interessante notar que há ainda um sistema de categorias de habilidades sociais educativas parentais que são testados e úteis no planejamento de intervenções sobre o tema em Del Prette e Del Prette (2008) e Rocha (2009).

Com professores, existem estudos mais antigos sobre o treinamento de habilidades sociais como o de Del Prette (1998) que, após 12 sessões completando dois meses de intervenção, observou-se melhora nos componentes não verbais de habilidades sociais, como expressão facial, entonação, humor e entusiasmo. Mais recentemente, Correa (2008) analisou modificações na relação professor-aluno com base em um treinamento em habilidades sociais em professores de uma escola pública de ensino fundamental. $\mathrm{O}$ treinamento favoreceu melhores ganhos nas contingências de interação professor-aluno com maior autocontrole da agressividade na interação social e assertividade. Em universitários, Del Prette e Del Prette (2003) enfatizam que houve uma redução na dificuldade de se executar uma série de condutas, como interromper conversas ao telefone, apresentar-se a alguém, cumprimentar desconhecidos, lidar com críticas, participar de conversação, elogiar quem faz algo bom, fazer pergunta a desconhecidos, fazer perguntas na escola ou no trabalho e pedir favor a um colega após intervenção. Vale ressaltar que esse foi o único estudo nacional a considerar tal aspecto, ou seja, habilidades a serem treinadas no processo de intervenção.

Em adolescentes, o artigo de Silva e Murta (2009) apresentam os resultados de um programa de intervenção de habilidades sociais vinculado ao programa de atenção integral à família. Os resultados indicaram uma melhora na liberdade de expressão e relatar sentimentos, problemas, fazer elogios, puxar conversa, pedir ajuda, dizer não e mesmo o desempenho acadêmico. No que se refere ao treinamento de habilidades sociais com crianças, Gonçalves e Murta (2008) evidenciaram aumento de condutas pró-sociais e mudanças positivas no autoconceito e julgamento dos pares a partir da análise do relato de crianças, de seus pares e pais. Os participantes também tenderam a ser mais aceitos na pós-intervenção pelos seus colegas de grupo. Também, no estudo de Faijão, Carneiro, Bruni, Montiel e Bartholomeu (2010), os resultados evidenciaram melhoras no sexo feminino quanto às habilidades de autoconhecimento, pensamento crítico, comunicação eficaz em comparação aos meninos. Estes se desenvolveram em ritmo mais lento que as meninas, não tendo tantas melhoras evidentes, apesar de terem exibido melhores resultados em relacionamento interpessoal que as meninas ao final da intervenção.

É interessante notar que a maior parte dos estudos nacionais que tratou das intervenções com habilidades sociais em crianças, por vezes, o faz indiretamente, via treinamento de pais ou professores, cuja literatura é mais vasta nos últimos seis anos no Brasil, e uma quantidade menor de investigações tratou de intervenções diretas com crianças. Além disso, a discussão sobre o nível de dificuldade das condutas a serem treinadas foi considerada somente em um dos artigos de Del Prette e Del Prette (2003) com uma amostra de universitários. Em crianças, o estudo de Del Prette \& Del Prette (2002) analisou as relações de indicadores de frequência e dificuldade de itens com medidas sociométricas em crianças, mas sem considerar esses elementos em um treinamento. Apesar disso, enfatizam que a medida de dificuldade das condutas tende a apresentar melhores características psicométricas na avaliação das 
habilidades sociais em crianças. Cabe mencionar que, neste estudo, não se está referindo ao parâmetro de dificuldade de itens derivado por Teoria de Resposta ao Item (TRI). A Teoria de Resposta ao Item permite fazer uma análise de cada item a partir de suas características e peculiaridades e, nesse caso, cada item se refere a um comportamento social específico. Além disso, os parâmetros estimados por esse modelo, tanto para itens como para as pessoas, são estáveis a partir do princípio de invariância dos parâmetros, possibilitando estimativas mais confiáveis das características dos itens e não dependentes das amostras.

O treinamento de habilidades sociais tem sido uma ferramenta amplamente utilizada no sentido de se operacionalizar pessoas quanto a condutas que são socialmente aceitas em um contexto dado, aumentando a probabilidade de interação entre os membros de um grupo qualquer (Bartholomeu, Carvalho, Silva, Miguel, \& Machado, 2011; Del Prette \& Del Prette, 2002). Ao se planejar um treinamento de habilidades sociais, frequentemente se recorre a instrumentos de avaliação que irão dar um panorama sobre que habilidades o indivíduo apresenta e quais estão deficitárias. No entanto, dentre as habilidades deficitárias, dificilmente se recorre a uma lógica de intervenção que considere o nível de dificuldade de se treinar certos comportamentos, uma vez que se desconhece o nível de dificuldade de cada tipo de conduta socialmente hábil (Cia \& Barham, 2009). Assim, ao se considerar tal variável no planejamento do treinamento de habilidades sociais, pode-se torná-la mais fácil a inserção de certos tipos de repertório de condutas sociais, já que se inicia pelos aspectos mais fáceis a serem treinados, passando aos mais difíceis gradativamente, o que tem uma implicação no sentido de favorecer uma maior segurança ao indivíduo que, ao iniciar por tarefas fáceis, sente-se mais confiante na execução do trabalho por conseguir aprender as etapas anteriores.

No artigo que descreve estudos iniciais para uma escala de avaliação das habilidades sociais em crianças, Bartholomeu, Silva e Montiel (2011) sugeriram uma hierarquia para o treinamento de habilidades sociais com o Teste de Habilidades Sociais para crianças e adolescentes em idade escolar (THAS-C) pelo modelo de Rasch da TRI e sugerem que o planejamento de intervenções com base na dificuldade dos itens pode facilitar o processo de aprendizagem dessas habilidades no sentido de inserção de novas condutas sociais no repertório do indivíduo. Todavia, os autores não sugerem maiores detalhes sobre a hierarquia de itens (condutas) por nível de dificuldade a serem trabalhados. O Standard Setting pode ser caracterizado pelo estabelecimento de um ou mais pontos de corte na escala de avaliação. Esse ponto de corte separa um nível de desempenho de outro, dividindo a distribuição do desempenho obtida pelas crianças em duas ou mais categorias distintas (Cizek \& Bunch, 2007).

No estudo de Bartholomeu et al. (2011), os autores indicam que as condutas relacionadas ao altruísmo e civilidade foram mais fáceis, conversação e desenvoltura social moderadas e assertividade mais difíceis. Assim, torna-se importante analisar se essa hierarquia seria mantida a partir de uma amostra maior e mais representativa do que a empregada pelos autores. Ao lado disso, a análise dos pontos de corte favoreceria uma melhor compreensão de que habilidades estão sendo efetivamente mudadas em intervenções de habilidades sociais quando planejadas pelo nível de dificuldade dos itens.

Nesse sentido, o presente estudo visa analisar se o planejamento de intervenção em habilidades sociais em crianças segundo o nível de dificuldade de cada conduta social pode facilitar o aprendizado delas. Com isso, primeiramente, foi estudado uma hierarquização dos itens do THAS-C a partir do modelo de Rasch para determinar quais seriam os pontos de corte na escala geral do THAS-C, visando identificar quais destes poderiam ser estabelecidos na medida proposta por Bartholomeu et al. (2011), sendo esse o objetivo do Estudo 1. Em seguida, foi realizada a análise da aplicação de um treinamento de habilidades sociais em um grupo de crianças, planejada conforme as avaliações do THAS-C (Estudo 2) e dos níveis de dificuldades dos itens e seus pontos de corte, visando testar a eficácia desse modelo de intervenção.

\section{Estudo 1}

\section{Método}

\section{Participantes}

Compuseram a amostra, 1.381 crianças e adolescentes de escolas públicas e particulares (cerca de 10\%) de cidades do interior do Estado de São Paulo, com idades entre 7 a 15 anos (média de idade $=9$ anos, $D P$ $=1,806)$. Em relação ao sexo, $52,1 \%$ eram do sexo masculino e 47,9\% eram do sexo feminino. Quanto ao ano escolar que frequentavam, esses foram de $2^{\circ}$ a $6^{\circ}$ e de $8^{\circ}$ ano, sendo que a maioria das crianças estava no $5^{\circ}$ ano $(32,4 \%)$. 


\section{Instrumento}

Teste de Habilidades Sociais em Crianças para o Ensino Fundamental (THAS-C) Bartholomeu, Silva \& Montiel, 2014)

Esse instrumento foi desenvolvido com base na insuficiência de pesquisas que atestassem as qualidades psicométricas de medidas de avaliação das habilidades sociais em crianças. Foram apresentados ao examinando 23 itens para serem respondidos quanto à frequência com que comete os comportamentos numa escala Likert de três pontos. As categorias de resposta foram Nunca, Às vezes e Sempre e foram atribuídos pontos de 1 a 3 respectivamente a cada uma delas na correção do instrumento que foram somados. Alguns itens são pontuados de forma inversa, já que a conduta socialmente hábil está definida no oposto do que a frase propõe (Bartholomeu et al., 2011).

Os resultados da análise preliminar feita por componentes principais e rotação Varimax sugeriram a estrutura de três fatores mais evidentes que explicaram $34,17 \%$ de variância. A primeira dimensão, Civilidade e altruísmo, abrange habilidades, como agradecer elogios, pedir desculpas, ajudar os amigos, elogiá-los, expressar sentimentos positivos aos pares, ser educado ao manifestar uma opinião. No segundo fator, Desenvoltura e autocontrole na interação social, seus indicadores devem ser invertidos para indicarem tais aspectos. As situações propostas sugerem situações negativas em que a criança é exposta a situações novas, desconhecidas ou que podem provocar constrangimento, como receber críticas, falar para a sala toda, encerrar uma conversa, apresentar-se a um grupo de desconhecidos ou perguntar-lhes algo. Finalmente, a terceira dimensão recebeu a denominação de Assertividade com enfrentamento. As habilidades propostas incluem demonstrar desagrado, defender seus direitos e opiniões, resistir à pressão do grupo, afirmando sua autoestima, sob o risco de uma reação indesejável por parte do interlocutor. Esses fatores foram similares aos sugeridos por Del Prette e Del Prette (2005).

\section{Procedimento}

A aplicação do instrumento ocorreu de forma coletiva, realizada nas salas de aulas dos alunos e somente àqueles cujos pais haviam autorizado previamente pela assinatura de um Termo de Consentimento Livre e Esclarecido sob o número do Comitê de Ética em Pesquisa 424-2010. Foi inicialmente explicado às crianças do que se tratava a pesquisa e como responder ao instrumento. As questões foram lidas uma a uma pelo pesquisador que aguardava a resposta antes de prosseguir a coleta. As crianças que não desejavam prosseguir tinham essa liberdade, sendo liberadas da aplicação.

\section{Resultados}

Os indicadores da escala de Habilidades Sociais para Alunos do Ensino Fundamental foram investigados quanto à sua adequação ao modelo de Rasch. Tal modelo considera somente o parâmetro de dificuldade dos itens para se estimar a adequação deles a uma escala, partindo do pressuposto da Teoria de Resposta ao Item. A precisão fornecida por esse modelo para os itens resultou em um coeficiente de 0,97 para os itens e 0,84 para as pessoas, o que favorece a interpretação de que as pessoas, ao responderem os itens, tendem a informar sobre as habilidades sociais com maior precisão. Todavia, os itens não foram capazes de avaliar as habilidades sociais denominadas verdadeiras das crianças com a mesma magnitude. O erro médio de medida para itens e pessoas, respectivamente foi de 0,04 (DP = $0,01)$ e $0,09(D P=0,01)$. Essas e outras informações constam da Tabela 1.

Tabela 1

Parâmetros de Ajuste de Itens e das Pessoas ( $N=1381)$

\begin{tabular}{lcccccccc}
\hline \multirow{2}{*}{ Parâmetros } & \multicolumn{3}{c}{ Itens } \\
\cline { 2 - 9 } & Dificuldade & Infit & Outfit & Erro & Habilidade & Infit & Outfit & Erro \\
\hline Média & 0,00 & 0,99 & 1,01 & 0,06 & 0,62 & 1,01 & 1,01 & 0,37 \\
DP & 0,41 & 0,26 & 0,28 & 0,00 & 1,01 & 0,52 & 0,52 & 0,06 \\
Máximo & 0,78 & 1,44 & 1,47 & 0,07 & 1,02 & 2,92 & 3,87 & 1,02 \\
Mínimo & $-0,56$ & 0,71 & 0,70 & 0,06 & $-3,55$ & 0,07 & 0,07 & 0,33 \\
\hline
\end{tabular}


Os itens forneceram média de outfit de 1,01 (DP = $5,6)$, indicando um bom ajuste dos itens nesse aspecto, de forma geral. Analisando mais detidamente esses dados, observa-se que a variação de outfit esteve entre os valores $1,47-0,70$, sugerindo que nem todos os itens estiveram dentro dos parâmetros de ajuste ao modelo. Nenhum item apresentou índices acima de 1,50 (Bond \& Fox, 2001). O procedimento de análise empregado para se interpretar as pontuações gerais brutas produzidas com o THAS-C (provenientes da soma dos escores nos fatores) foi o uso do mapa do construto ou mapa de respostas esperadas, baseado na Teoria de Resposta ao Item, mais especificamente, no modelo de Rasch.

Por esse mapa, pode-se investigar as relações entre os níveis de habilidades e o nível de concordância com cada comportamento específico, favorecendo uma melhor compreensão do que cada pessoa em cada um dos níveis da escala é capaz de fazer em termos de comportamento social. Assim, podem-se dividir faixas de escores, estabelecendo pontos de corte na medida em que indiquem o que cada nível na escala sugere em termos de habilidades sociais, o que é denominado na literatura específica de standard setting. Analisando o índice de separação (um modo de precisão das medidas pelo modelo de Rasch) sugeriu que esse teste separa no máximo três grupos significativamente (Separation $=$ 2,30). Considerando esse fato e analisando-se a Figura 1 em questão, ao se traçar uma linha de alto a baixo nesse gráfico e analisar o cruzamento desta nos escores esperados de cada item, verifica-se o padrão de comportamentos esperados para cada nível de corte estabelecido e optou-se pela definição de dois pontos de corte na medida $-0,9$ e 1,74, restando três faixas na escala, sendo elas, baixa habilidade social, habilidade social mediana e alta habilidade social.

Esses pontos de corte foram estabelecidos em logit (medida fornecida pelo programa winsteps pelo modelo de Rasch), mas tem uma representação direta na escala bruta do teste. Assim, após a soma dos escores nos três fatores, o primeiro ponto de corte que separa a faixa de baixa habilidade da média é 15 , e o segundo ponto de corte que separa habilidade média de superior é 37. Nesse contexto, a faixa de baixa habilidade social é dada com escores totais brutos abaixo

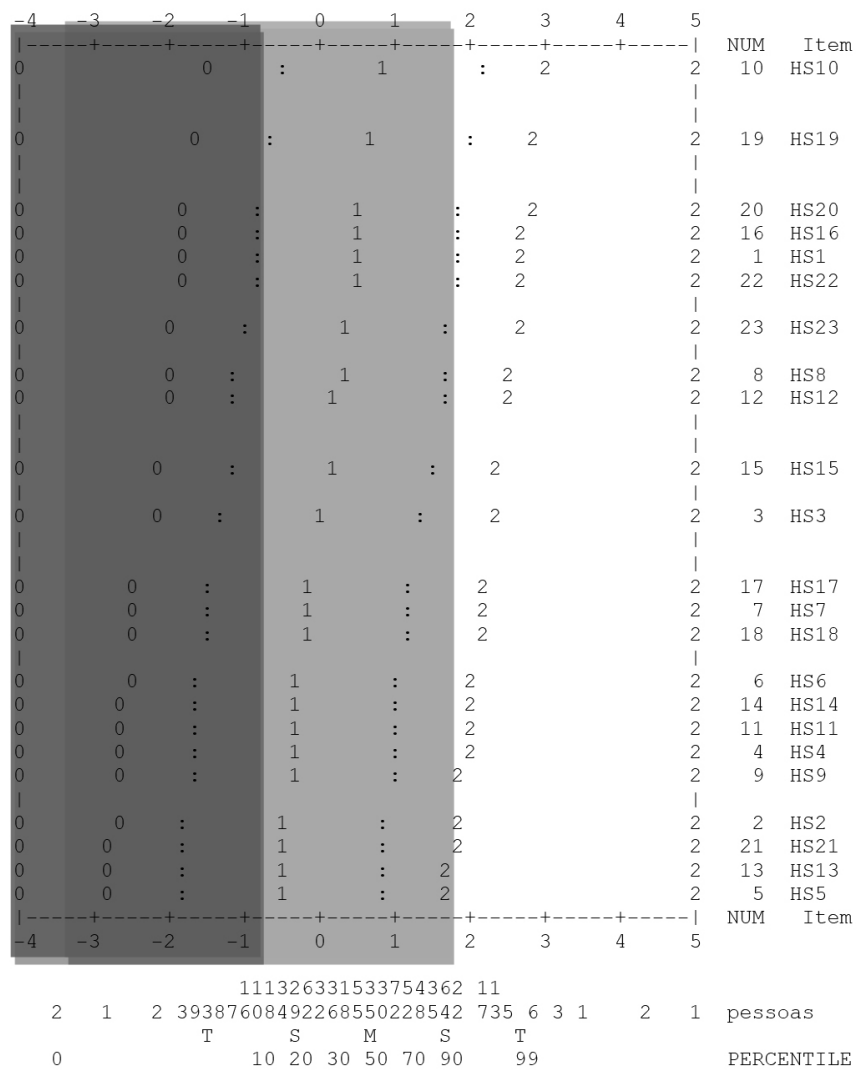

Figura 1. Itens do THAS-C com dificuldades fáceis e medianas. 
de 15 (parte azul da Figura). Analisando o padrão de respostas de pessoas nessa faixa, identifica-se uma baixa frequência da maior parte dos comportamentos e uma ausência de certas condutas, as mais difíceis de serem executadas conforme essa escala. Assim, essas pessoas tendem a executar algumas vezes somente a maior parte dos comportamentos descritos nos itens do THAS-C com dificuldades fáceis e medianas e a nunca conseguirem entrar na brincadeira quando zombam delas, ter vergonha em situações de exposição, dificuldades de encerrar conversas, sentir raiva quando criticadas justamente e a não expressar desagrado aos colegas.

A faixa de habilidade social mediana (faixa verde na Figura 1) caracteriza pessoas com pontuação $16 \mathrm{e}$ 36 na escala bruta e que tendem a executar comportamentos sociais, em sua maioria algumas vezes, mas que tendem a ter alta concordância com condutas mais fáceis de serem emitidas no contexto social, como pedir desculpas, elogiar amigos, ajudá-los, agradecer elogios, dentre outros, em sua maioria, relacionados às regras de civilidade e condutas altruístas. Pode ainda haver concordância mediana também em alguns dos comportamentos mais difíceis de serem executados, como encerrar conversas e não sentir vergonha em situações de exposição. Finalmente, a faixa de alta habilidade social (pontuação bruta acima de 37) caracteriza pessoas na faixa branca da Figura 1 e que tendem a executar todas as condutas descritas nesse teste com frequência alta, sendo que, nas questões mais difíceis, podem até apresentar em incidência mediana (comportamentos como habilidades para encerrar conversas e entrar na brincadeira quando zombam dele), mas as mais fáceis, como as condutas altruístas e cíveis, ser assertivo e defender seus direitos, mesmo quando o risco de reação indesejável pelo interlocutor é alto, tendem a fazer com muita frequência.
Em conseguinte foram testadas as diferenças na medida de habilidade social geral entre os grupos formados com base no mapa de itens para identificar se os níveis de habilidade social das pessoas diferiram entre si. Essa análise foi feita pela prova Anova e revelou diferenças significativas (Tabela 2). A prova de Tukey também indicou uma separação dos três grupos separados com base no critério do nível de habilidade social das pessoas em questão.

\section{Estudo 2}

Estabelecidos os pontos de corte na medida, procurou-se trabalhar o treinamento de habilidades sociais em crianças, como estratégia de auxílio na solução de problemas e conflitos interpessoais na escola. Em outros termos, visa testar a eficácia do treinamento em habilidades sociais a partir da hierarquização das condutas pelo modelo de Rasch, considerando os pontos de corte de habilidades sociais alta, média e baixa.

\section{Método}

\section{Participantes}

A pesquisa de campo foi aplicada em um grupo de 36 alunos do $4^{\circ}$ ano do ensino fundamental de ambos os sexos com $50 \%$ do sexo masculino, todas provenientes de escolas públicas de uma cidade do interior do estado de São Paulo. Todas as crianças tinham 10 anos de idade e apresentavam queixas de problemas de conduta em sala de aula e dificuldades de aprendizagem, segundo a opinião dos professores.

\section{Procedimentos e Materiais}

O trabalho foi realizado com todos os alunos em uma sala, sendo que eles foram divididos em três grupos de 12 crianças mas sem o objetivo de estabelecer

Tabela 2

Prova de Tukey, Valor de F e Nivel de Significância para a Medida de Habilidade Social Geral e as Faixas Etárias por Idade

\begin{tabular}{|c|c|c|c|c|}
\hline \multicolumn{5}{|l|}{$F=481,70, p=0,000$} \\
\hline \multirow{2}{*}{ Faixas separadas com base no critério } & \multirow{2}{*}{$\mathrm{N}$} & \multicolumn{3}{|c|}{ Subgrupo para alfa $=0,05$} \\
\hline & & 1 & 2 & 3 \\
\hline Baixa habilidade social & 65 & 12,14 & & \\
\hline Habilidade social mediana & 680 & & 28,36 & \\
\hline Alta habilidade social & 44 & & & 38,91 \\
\hline Sig. & & 1,00 & 1,00 & 1,00 \\
\hline
\end{tabular}


grupos de controle, já que optou-se por manter grupos menores na intervenção e todos (dadas as suas dificuldades) deveriam passar por ela conforme a solicitação da escola. Esse espaço estava separado do local de aula. A intervenção totalizou 12 sessões realizadas semanalmente, com duração de duas horas cada. A avaliação do THAS-C foi realizada no início e ao final desse processo. Foi aplicado um treinamento de estratégias para resolução de conflitos a partir de dinâmicas de grupo estruturadas com base no nível de dificuldade dos itens focadas na questão de resolução de conflitos interpessoais. Os temas das dinâmicas tiveram como objetivo demonstrar às crianças a importância que a pessoa tem em sua vida, incentivando principalmente a empatia. Cada dinâmica foi executada por duas sessões. A Tabela 3 apresenta uma síntese das atividades realizadas, valendo ressaltar que as dinâmicas e elementos a serem treinados foram estabelecidos após a primeira avaliação e identificação do nível de habilidades sociais da amostra em questão.

\section{Resultados}

A classificação média das crianças do grupo em questão foi "repertório mediano de habilidades sociais". Assim, baseou-se parte das intervenções nos resultados considerados como medianos pela hierarquização das condutas extraídas pelo THAS-C. Todavia, $30 \%$ das crianças apresentaram déficits de habilidades sociais (Faixa 1), cujo foco principal são os sentimentos de vergonha e raiva na situação de interação, além da não execução de condutas assertivas, como cíveis e de desenvoltura social. As crianças nessa faixa, além de carecerem de um repertório adequado de habilidades

Tabela 3

Procedimento de Sessões, Objetivos e Elementos Treinados na Intervenção de Habilidades Sociais com Crianças Baseado na Dificuldade das Condutas

\begin{tabular}{llll}
\hline Encontro/Atividade & Objetivo & Elemento de HS. treinado & $\begin{array}{l}\text { Faixa de dificuldade } \\
\text { (THAS-C) }\end{array}$ \\
\hline $\begin{array}{l}\text { Início de Atividades/ } \\
\text { Apresentação/ } \\
\text { Avaliação THAS }\end{array}$ & $\begin{array}{l}\text { Conhecer o grupo, separar o } \\
\text { grupo e avaliar as habilidades } \\
\text { sociais. }\end{array}$ & & \\
\hline $\begin{array}{l}\text { Dinâmica: Bola } \\
\text { imaginária }\end{array}$ & $\begin{array}{l}\text { Promover aceitação de si e dos } \\
\text { outros, respeitar diferenças. }\end{array}$ & $\begin{array}{l}\text { Civilidade e altruísmo, } \\
\text { sentimentos de vergonha e raiva } \\
\text { na interação social. }\end{array}$ & \\
\hline $\begin{array}{l}\text { Dinâmica: Passa ou } \\
\text { repassa. }\end{array}$ & $\begin{array}{l}\text { Promover iniciação de } \\
\text { conversação, perceber o } \\
\text { sentimento do outro e ajudar os }\end{array}$ & $\begin{array}{l}\text { Agradecer, pedir ajuda, oferecer } \\
\text { ajuda, sentimentos de vergonha }\end{array}$ & 1,2 \\
& e raiva na interação (altruísmo, & \\
civilidade). & \\
\hline Dinâmica: Gato e rato como pedir ajuda. & $\begin{array}{l}\text { Estimular ajuda mútua, } \\
\text { tomada de decisão em relações }\end{array}$ & $\begin{array}{l}\text { Expor sentimentos, iniciar } \\
\text { conversação (altruísmo, } \\
\text { habilidades de conversação). }\end{array}$ & 2 \\
& $\begin{array}{l}\text { interpessoais e expressão de } \\
\text { sentimentos e habilidades de } \\
\text { conversação e negociação. }\end{array}$ & & \\
& $\begin{array}{l}\text { Estimular a autoexposição } \\
\text { e habilidades de encerrar } \\
\text { conversas e tolerar frustrações. }\end{array}$ & $\begin{array}{l}\text { Habilidades de conversação e } \\
\text { exposição. }\end{array}$ & 2 \\
\hline $\begin{array}{l}\text { Dinâmica: Hipnose } \\
\text { com as mãos }\end{array}$ & $\begin{array}{l}\text { Trabalhar expressão e recepção } \\
\text { de sentimentos positivos e } \\
\text { negativos. Empatia. }\end{array}$ & $\begin{array}{l}\text { Altruísmo, civilidade, } \\
\text { conversação. }\end{array}$ \\
\hline $\begin{array}{l}\text { Dinâmica: Avaliação } \\
\text { criativa }\end{array}$ & $\begin{array}{l}\text { Obter percepções sobre o } \\
\text { processo de intervenção e } \\
\text { reavaliar. }\end{array}$ & & \\
\hline $\begin{array}{l}\text { Encerramento/ } \\
\text { Avaliação THAS }\end{array}$ & & \\
\hline
\end{tabular}


sociais, tendem a sentir raiva e vergonha nas situações de interação. Nesse sentido, as primeiras dinâmicas também envolveram estratégias de manejo de componentes emocionais, como técnicas de controle de raiva e técnicas focadas no controle de pensamentos, visando um cuidado com a vergonha na interação social. De fato, após a intervenção, somente $10 \%$ permaneceu na Faixa 1, e $90 \%$ ficaram na Faixa 2, diferença que foi estatisticamente significativa $(p=0,017)$. É interessante notar que não houve pessoas na Faixa 3 e a intervenção não foi focada nas condutas de transição da Faixa 2 para a 3 o que pode explicar em partes esse fato. A intervenção foi focada nos déficits mais agudos e em retirar crianças da Faixa 1, bem como estimular crianças da Faixa 2. De fato, a média de desempenho nessa faixa aumentou e chegou próxima à Faixa 3, sugerindo que a continuidade do processo de intervenção com mudanças mais específicas nesse nível poderiam ser benéficas e agregar mais ao repertório das crianças em questão. A análise dos resultados de habilidades sociais antes e depois do treinamento em solução de conflitos interpessoais por fator do THAS-C foi feita pela prova de Wilcoxon com nível de significância de 0,05 .

Por esses dados, observa-se que o altruísmo das crianças tendeu a aumentar após a intervenção em solução de conflitos, bem como as habilidades sociais gerais $(p=0,002 ; p=0,005$, respectivamente; diferença de média de 3,22 em altruísmo e 4,34 em habilidade social geral entre pré e pós-teste). Vale notar que, apesar de não serem diferenças significativas, a assertividade das crianças tendeu a diminuir levemente e a desenvoltura a aumentar. Assim, pode-se dizer que o treinamento de solução de conflitos tendeu a melhorar o altruísmo dessas crianças, tornando-as mais capazes de agradecer a elogios, pedir desculpas, ajudar os amigos, elogiá-los, expressar sentimentos positivos, ser educado ao manifestar uma opinião, dentre outras condutas. O treinamento tendeu a melhorá-las nesse sentido e um investimento nessa direção pode favorecer incrementos ainda maiores em particular. De fato, a sequência do planejamento dessa intervenção deve focar nos aspectos da desenvoltura social e assertividade como sugere esse teste.

\section{Discussão Geral}

Este trabalho teve como objetivo testar a possibilidade de se aplicar um treinamento de habilidades sociais em crianças baseado na hierarquização por nível de dificuldade dos comportamentos sociais, realizados pelo modelo de Rasch e utilizando o THAS-C para a avaliação dos comportamentos sociais em crianças. Para isso, foi estabelecida uma hierarquização dos itens do instrumento, e obtidos pontos de corte para facilitar a classificação do tipo de repertório de habilidades sociais das crianças, bem como avaliar a mudança com a intervenção realizada no Estudo 2. Cabe mencionar que Bartholomeu et al. (2011) em um estudo preliminar com o THAS-C sugeriram que pontos de corte nessa medida poderiam ser feitos, já que os fatores evidenciados pela análise fatorial foram separados, aparentemente pelo nível de dificuldade dos itens sendo que o altruísmo e civilidade foi o mais fácil; moderado, conversação e desenvoltura social; e difícil, assertividade e enfrentamento, o que se confirma no presente estudo. A quantidade de desajustes dos itens e pessoas ao modelo de Rasch na amostra do presente trabalho foi menor, indicando um melhor ajuste dessa escala, apesar de ela ainda aferir níveis médios de habilidade social no geral, podendo ser incluídos novos itens para aferir habilidades sociais mais difíceis de serem executadas pelas crianças. Assim, uma continuidade das pesquisas poderia incluir esse teste em formato adaptativo ou mesmo a construção de itens mais difíceis para serem incluídos nessa escala. O presente trabalho também implementa o primeiro estudo do THAS-C, na medida que sugere padrões de resposta característicos de crianças altamente habilidosas socialmente e pouco habilidosas.

Do ponto de vista da intervenção, é interessante notar que o modelo de Rasch trabalha com predições e que, em dados ajustados ao modelo (como foi o caso neste trabalho), implica em uma progressão do nível de habilidade das pessoas em razão da dificuldade dos itens de uma escala, logo, se o treinamento dessas habilidades fosse efetivo e a habilidade aumentada, uma maior concordância com itens mais difíceis seria esperada. Assim, os resultados do Estudo 2 confirmam a eficácia do modelo de Rasch em estabelecer as predições, já que o treinamento focou no ínterim entre os níveis 1 e $2 \mathrm{em}$ que a maioria das pessoas se situava. Achou-se pouco produtivo treinar habilidades mais elevadas no grupo (nível 3), considerando que havia ainda cerca de um quarto do grupo com problemas no nível 1 e que essas pessoas poderiam ficar ainda mais defasadas caso o treinamento fosse direto para o nível mais elaborado por não terem as habilidades medianas estabelecidas em seu repertório.

O estudo de Del Prette e Del Prette (2003) evidenciou redução da dificuldade de uma série de 
condutas sociais em universitários a partir de um treinamento com módulos teóricos e práticos, como apresentar-se a alguém, cumprimentar desconhecidos, lidar com críticas, participar de conversação, elogiar, fazer perguntas, pedir favores, dentre outros. Muitas dessas condutas envolvem também habilidades de conversação e desenvoltura social, altruísmo e mesmo assertividade, e os autores enfatizam os benefícios de módulos teóricos no treinamento de habilidades sociais com essa população. Nesse sentido, apesar de o nível de dificuldade não ter sido estimado a partir de um procedimento de Standard Setting, o uso desse elemento como facilitador do tratamento demonstrou eficácia. As habilidades treinadas abrangem condutas de mais de um dos níveis estimados em crianças pelo THAS-C. Assim, apesar de as medidas não estarem equalizadas (THAS-C e IHS avaliadas em uma mesma escala) e não se poder afirmar se aferem níveis de habilidades empíricos semelhantes, pode-se indicar uma direção de novas investigações visando caracterizar níveis de dificuldade de execução de condutas sociais para cada idade e/ou nível de escolaridade.

Observou-se, ao final do treinamento de condutas do nível 1 e 2 , um avanço das pessoas que estavam no nível 1 para o nível 2 e de pessoas do nível 2 cujos padrões de concordância ainda eram medianos para padrões de concordância altos, já adentrando no nível 3 (mais difícil). As condutas que melhoraram referiram-se, em sua maioria a condutas pró-sociais, altruistas e de conversação, o que se coaduna com pesquisas de Gonçalves e Murta (2008) e Faijão et al., (2010) em que evidenciaram melhoras, sobretudo em condutas pró-sociais e de comunicação em crianças.

Aparentemente, quando se trata de condutas sociais, estas parecem ser os primeiros pontos que frequentemente melhoram não só em crianças como em universitários (Del Prette \& Del Prette, 2003), adolescentes (Silva \& Murta, 2009) e mesmo em pais de crianças com problemas de conduta (Pinheiro et al., 2006) e demonstra indicar que, mesmo em outros treinamentos que não foram sistematizados com base na hierarquia de dificuldade das condutas sociais, as que são mais fáceis de serem treinadas são as frequentemente as que mais melhoram. Nesse sentido, é oportuno mencionar que valeria à pena analisar isso mais detidamente ao se hierarquizar as habilidades sociais em outras etapas do desenvolvimento, como adolescentes e adultos, para se identificar ou não se essa seria uma forma adequada de se planejar os treinamentos nesses grupos. Ainda cabe mencionar que, ao se empregar o modelo de Rasch que partilha do princípio da TRI de invariância de parâmetros, seria esperado que, em outras amostras de crianças, essas condutas se mantivessem com níveis de dificuldade semelhantes. Apesar disso, esse fato há que ser mais devidamente investigado, já que pelas teorias de habilidades sociais estas mudam em razão do contexto, em decorrência de expectativas e valores. Assim é plausível apontar que um mesmo comportamento social adequado em um contexto pode não o ser em outro. Esse fato convida a novos estudos e investigações. Ainda, novas pesquisas poderiam ser feitas no sentido de se estabelecer níveis de dificuldade em diferentes etapas da vida e contextos para as condutas socialmente habilidosas que, por definição, seriam esperadas que fossem distintos.

\section{Considerações Finais}

Apesar de se ter demonstrado a eficácia do modelo de Rasch como auxiliar na avaliação e intervenção com as habilidades sociais no sentido de se aferir os níveis de dificuldade de cada comportamento social no planejamento de intervenções, não se pode afirmar que esse tipo de intervenção baseada na hierarquização de dificuldade de condutas a serem treinadas seja mais eficaz que outros tipos de intervenção em habilidades sociais, já que não se testou outras formas paralelamente a essa visando compará-las. Também se empregou delineamento pré-experimental no Estudo 2, o que não permite fazer afirmações causais nesse sentido. Assim, sugerem-se novas investigações com delineamento experimental e quase experimental para avaliar com maior controle e rigor os efeitos desse tipo de intervenção, bem como comparar com outros tipos. Murta (2005) indicou em seu estudo de revisão uma carência deste tipo de pesquisa no que se refere ao treinamento de habilidades sociais, reiterando tal necessidade.

Outra questão que procede dos resultados ora obtidos é: será que o aprendizado é melhor e mais consistente com essa modalidade de treinamento do que treinar habilidades variadas, já que o grupo com habilidades moderadas para altas não progrediu? Será que as crianças mais habilidosas não progrediriam por estarem em um nível abaixo da escala e ainda serem condutas muito difíceis de executarem dado seu repertório comportamental? Finalmente, uma quantidade maior de sessões e a continuidade no planejamento do treinamento de habilidades sociais incluindo mais condutas da faixa 3 de dificuldade do THAS-C seria útil para verificar se as mudanças seguem ocorrendo. Ainda vale 
ressaltar que o procedimento de avaliação baseado no Rasch Item Mapping Method demonstrou eficácia quanto à indicação ao treinamento das condutas socialmente hábeis e deve ser explorado, bem como ampliado seu uso a partir da inclusão de novos itens na escala para aferir níveis mais elevados de habilidades sociais.

\section{Referências}

Bartholomeu, D., Carvalho, L. F., Silva, M. D., Miguel, F. K., \& Machado, A. A. (2011). Aceitação e rejeição entre pares e habilidades sociais em universitários. Estudos de Psicologia (Natal), 16(2), 155-162. Recuperado de http://www.scielo.br/pdf/epsic/v16n2/ v16n2a06.pdf

Bartholomeu, D., Montiel, J. M., \& Pessotto, F. (2011). Sociometria e habilidades sociais em alunos do ensino médio. Estudos Interdisciplinares em Psicologia, 2(2), 211-228. Recuperado de http://www. uel.br/revistas/uel/index.php/eip/article/ viewFile/10928/9553

Bartholomeu, D., Silva, M. C. R., \& Montiel, J. M. (2014). Teste de Habilidades Sociais em Crianças e Adolescentes (THAS-C). Editora Memnon. São Paulo.

Bartholomeu, D., Silva, M. C. R., \& Montiel, J. M. (2011). Teste de habilidades sociais para crianças: Evidências psicométricas de uma versão inicial. Psico-USF, 16(1) 33-43. doi: 10.1590/ S1413-82712011000100005

Bolsoni-Silva, A. T., Silveira, F. F., \& Ribeiro, D. C. (2008). Avaliação dos efeitos de uma intervenção com mães/cuidadoras: Contribuições do treinamento em habilidades sociais. Contextos Clínicos, 1, 19-27. Recuperado de http://pepsic.bvsalud.org/ pdf/cclin/v1n1/v1n1a03.pdf

Bolsoni-Silva, A. T., Salina-Brandão, A., Versuti-Stoque, F. M., \& Rosin-Pinola, A. R. (2008). Avaliação de um programa de intervenção de habilidades sociais educativas parentais: Um estudo-piloto. Psicologia: ciência e profissão, 28(1), 18-33. doi: 10.1590/ S1414-98932008000100003

Bond, T. G. \& Fox, C. M. (2001) Applying the Rasch model: Fundamental measurement in the human sciences. Mahwah, N.J.: Erlbaum.

Cia, F., \& Barham, E. J. (2009). Repertório de habilidades sociais, problemas de comportamento, autoconceito e desempenho acadêmico de crianças no início da escolarização. Estudos de Psicologia, 26, 45-55. doi: 10.1590/S0103-166X2009000100005

Cizek, G. J., \& Bunch, M. B. (2007). Standard setting: A guide to establishing and evaluating performance standards on tests. Thousand Oaks: SAGE Publications.

Corrêa, C. I. M. (2008). Habilidades sociais e educação: Programa de intervenção para professores de uma escola pública (Tese de doutorado). Universidade Estadual Paulista, Brasil. 140p.

Del Prette, A., \& Del Prette, Z. A. P. (2003). No contexto da travessia para o ambiente de trabalho: Treinamento de habilidades sociais com universitários. Estudos de Psicologia (Natal), 8(3), 413-420. doi: 10.1590/S1413-294X2003000300008

Del Prette, A. (1998). Efeitos de uma intervenção sobre a topografia das habilidades sociais de professores. Psicologia Escolar e Educacional, 2(1), 11-22. doi: 10.1590/S1413-85571998000100002

Del Prette, Z. A. P., Barreto, S. O., \& Freitas, L. C. (2011). Habilidades sociais na comorbidade entre dificuldades de aprendizagem e problemas de comportamento: Uma avaliação multimodal. Psico, 42(4), 503-510. Recuperado de http://revistaseletronicas.pucrs.br/ojs/index.php/revistapsico/ article/view/7593

Del Prette, Z. A. P. \& Del Prette, A. (2005). Habilidades sociais na infância: teoria e prática. Petrópolis: Vozes.

Del Prette, Z. A. P., \& Del Prette, A. (2008). Um sistema de categorias de habilidades sociais educativas. Paidéia (Ribeirão Preto), 18(41), 517-530. doi: 10.1590/ S0103-863X2008000300008

Del Prette, Z. A., \& Del Prette, A. (2002). Avaliação de habilidades sociais de crianças com um inventário multimídia: Indicadores sociométricos associados à frequência versus dificuldade. Psicologia em Estudo, 7(1), 61-73. doi: 10.1590/S1413-73722002000100009

Faijão, W., Carneiro, G. R. S., Bruni, A. R., Montiel, J. M., \& Bartholomeu, D. (2010). Aplicação de um treinamento de habilidades sociais em crianças do ensino fundamental. Encontro: Revista de Psicologia, 13(19), 36-42 Recuperado de http://www.scielo. br/pdf/prc/v18n2/27480.pdf

Freitas, L. C., \& Del Prette, Z. A. P. (2010). Validade de construto do sistema de avaliação de habilidades sociais para crianças brasileiras com deficiência 
intelectual. Interamerican Journal of Psychology, 44(2), 312-320. Recuperado de http://www.redalyc.org/ articulo.oa?id $=28420641012$

Gonçalves, E. S., \& Murta, S. G. (2008). Avaliação dos efeitos de uma modalidade de treinamento de habilidades sociais para crianças. Psicologia: Reflexão e Crítica, 21(3), 430-436. doi: 10.1590/ S0102-79722008000300011

Molina, R. C. M., \& Del Prette, Z. A. P. (2006). Funcionalidade da relação entre habilidades sociais e dificuldades de aprendizagem. Psico-USF, 11(1), 53-63. doi: 10.1590/S1413-82712006000100007

Murta, S. G. (2005). Aplicações do treinamento em habilidades sociais: Análise da produção nacional. Psicologia: Reflexão e Crítica, 18(2), 283-291. Recuperado de http://www.scielo.br/pdf/prc/ v18n2/27480.pdf
Pinheiro, M. I. S, Haase, V. G., Del Prette, A., Amarante, C. L. D., \& Del Prette, Z. A. P. (2006). Treinamento de habilidades sociais educativas para pais de crianças com problemas de comportamento. Psicologia: Reflexão e Crítica, 19(3), 407-414. doi: 10.1590/ S0102-79722006000300009

Rocha, M. M. (2009). Programa de habilidades sociais com pais: Efeitos sobre o desempenho social e acadêmico de filhos com TDAH (Tese de doutorado). Universidade de São Carlos, Brasil. 235p.

Silva, M. P., \& Murta, S. G. (2009). Treinamento de habilidades sociais para adolescentes: Uma experiência no programa de atenção integral à família (PAIF). Psicologia: Reflexão e Crítica, 22(1), 136-143. doi: 10.1590/S0102-79722009000100018

Recebido em: 07/07/2014

Primeira reformulação em: 30/03/2015

Segunda reformulação em: 02/04/2015

Aprovado em: 07/04/2015

Sobre os autores:

José Maria Montiel é psicólogo, especialista em Diagnóstico e Triagem, mestre e doutor em Avaliação Psicológica pela Universidade São Francisco, atualmente é coordenador do Núcleo de Pesquisa em Saúde e Desempenho Humano e professor do Programa de Pós-Graduação Strictu Sensu em Psicologia Educacional - Centro Universitário FIEO - UNIFIEO - São Paulo.

E-mail:montieljm@hotmail.com

Daniel Bartholomeu é psicólogo, mestre, doutor em Avaliação Psicológica pela Universidade São Francisco, colaborador do Laboratório de Pesquisa em Psicologia do Esporte (Lepespe) e, atualmente, é professor Programa de Pós-Graduação Strictu Sensu em Psicologia Educacional - Centro Universitário FIEO - UNIFIEO - São Paulo. E-mail:d_bartholomeu@yahoo.com.br

Contato com os autores:

José Maria Montiel

Centro Universitário FIEO - UNIFIEO/SP

Avenida Franz Voegelli, 300 Vila Yara - Bl. Prata

CEP: 06020-190

E-mail:montielim@hotmail.com

Psico-USF, Bragança Paulista, v. 21, n. 1, p. 37-47, jan./abr. 2016 
